Carbon nanotubes in neural interfacing applications

This article has been downloaded from IOPscience. Please scroll down to see the full text article.

2011 J. Neural Eng. 8011001

(http://iopscience.iop.org/1741-2552/8/1/011001)

View the table of contents for this issue, or go to the journal homepage for more

Download details:

IP Address: 141.211.173.82

The article was downloaded on 06/04/2012 at 16:55

Please note that terms and conditions apply. 


\title{
Carbon nanotubes in neural interfacing applications
}

\author{
Christopher M Voge and Jan P Stegemann ${ }^{1}$ \\ Department of Biomedical Engineering, University of Michigan, Ann Arbor, MI, USA \\ E-mail: jpsteg@umich.edu
}

Received 5 September 2010

Accepted for publication 10 December 2010

Published 19 January 2011

Online at stacks.iop.org/JNE/8/011001

\begin{abstract}
Carbon nanotubes (CNT) are remarkable materials with a simple and inert molecular structure that gives rise to a range of potentially valuable physical and electronic properties, including high aspect ratio, high mechanical strength and excellent electrical conductivity. This review summarizes recent research on the application of CNT-based materials to study and control cells of the nervous system. It includes the use of CNT as cell culture substrates, to create patterned surfaces and to study cell-matrix interactions. It also summarizes recent investigations of CNT toxicity, particularly as related to neural cells. The application of CNT-based materials to directing the differentiation of progenitor and stem cells toward neural lineages is also discussed. The emphasis is on how CNT surface chemistry and nanotopography can be altered, and how such changes can affect neural cell function. This knowledge can be applied to creating improved neural interfaces and devices, as well as providing new approaches to neural tissue engineering and regeneration.
\end{abstract}

(Some figures in this article are in colour only in the electronic version)

\section{Introduction}

Over the last two decades, carbon nanotubes (CNT) have been at the forefront of advances in a diverse range of disciplines, from materials science [1] to electrical engineering [2] and biomedicine [3]. CNT have a highly ordered and stable molecular structure, giving them remarkable properties, including very high tensile strengths, aspect ratios and electrical conductivity [4]. Figure 1 shows the general geometry and lattice structure of both single-walled carbon nanotubes (SWNT) and multi-walled carbon nanotubes (MWNT). Initially, the use of CNT in biological systems was limited by poor solubility and the presence of toxic metallic impurities introduced by the fabrication process. More recently, advances in synthesis, purification and functionalization have improved the purity and biocompatibility of CNT materials [5-8], and they currently are being actively investigated as components of advanced

\footnotetext{
1 Author to whom any correspondence should be addressed.
}

biosensors, implant coatings and composite biomaterials [9-11].

The field of neural engineering has been energized by the design and fabrication of nanomaterials and nanodevices that can be used to study neural function. CNT offer important physical, chemical and electronic advantages that make them well suited for this purpose. The nanoscale roughness and porosity created by CNT materials offer a unique neuronal interface that can be beneficial for recording fine neural signals in dynamic systems [12-14]. The surface of CNT can be functionalized with different polymers or other bioactive molecules to improve biocompatibility and bioactivity. Figure 2 shows a schematic of how the CNT surface can be covalently functionalized, noncovalently wrapped or decorated with bioactive molecules. Such changes or additions to the carbon lattice can vary the reactivity of the CNT in specific ways [15, 16]. Depending on the conformation of the carbon lattice, CNT can also exhibit a range of electrical states, from metallic to semiconducting. Finally, the 

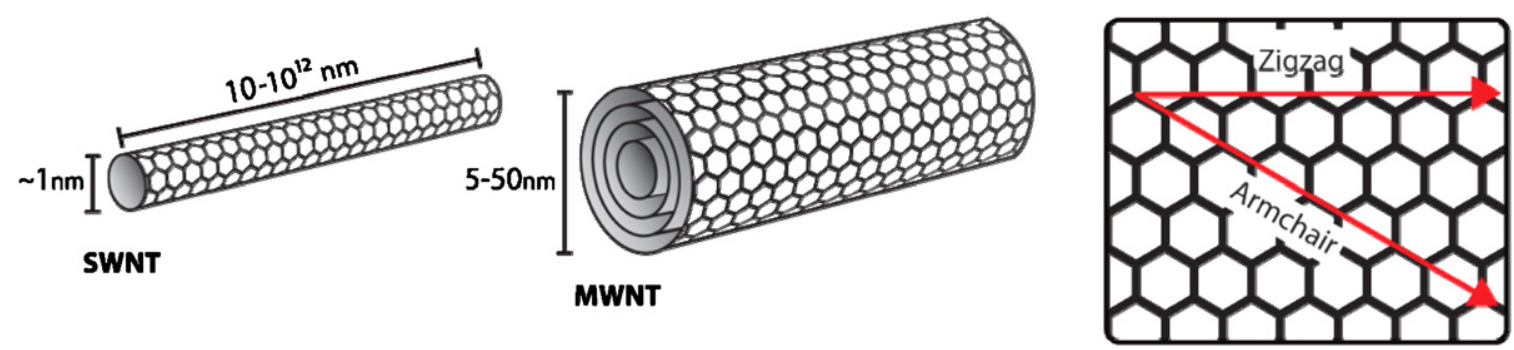

Figure 1. CNT can be considered as sheets of graphene rolled along a single side into a hollow tubular structure. Single-walled nanotubes (SWNT) have diameters close to $1 \mathrm{~nm}$ with a length that can be $10^{12}$ times longer. Multi-walled nanotubes (MWNT) consist of a number of concentric carbon tubes and vary correspondingly in diameter. The electronic structure of a CNT is highly dependent on how the hexagonal carbon lattice is oriented between zigzag (metallic and semiconducting) and armchair (metallic) configurations.

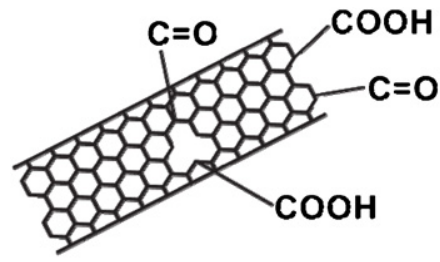

Covalent

Modification

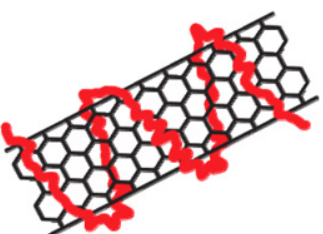

Noncovalent Wrapping

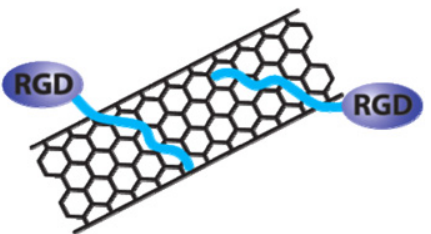

Ligand

Attachment

Figure 2. Functionalization of the CNT surface can be used to improve dispersion, change surface charge and deliver specific molecules. Covalent modification involves chemically modifying the lattice by attaching specific chemical groups at defect sites. Non-covalent wrapping methods rely on hydrophobic interactions and high shear mixing to coat CNT in appropriately chosen polymers and surfactants. Ligand attachment is a covalent or non-covalent modification of the nanotube surface to present specific biologically active moieties, including drugs or oligopeptides.

high aspect ratio of CNT can promote anisotropic electrical propagation and is potentially important for aligning cells or inducing migration [17-19]. These properties give CNT high potential for studying and controlling biological phenomena in neuroscience.

The use of CNT in medicine has raised interest in their potential toxicity. The effect of CNT on living systems has become a highly active area of research over the last decade [20, 21]; however, there is still no broad consensus on what features of nanoparticles make them more or less biocompatible. It should be noted that nanoparticles are being developed for many cell- and tissue-contacting applications, and therefore biocompatibility is necessarily an important issue. Some of these materials, including CNT, are rapidly approaching targeted clinical use [22, 23] and it is highly likely that CNT will find medical uses. However it has become clear that it is impossible to broadly classify CNT as 'nontoxic' or 'toxic', since their effects on cells are highly dependent on the application. In this respect they are very similar to most other molecules and materials that are used in medicine: their efficacy and side effects depend greatly on the dose, mode of administration and type of exposure. In spite of these concerns, it is likely that CNT will find utility as experimental tools in neuroscience and in addition will form the basis of new technologies in neuromedicine.

This review examines how CNT have advanced the fields of neurobiology and neural engineering. Particular emphasis is placed on how neural cells interact with CNT, including an examination of cytotoxicity and CNT affects on stem cell differentiation. The first section of this paper focuses on how two-dimensional (2D) substrates incorporating CNT have been used for neural cell culture, and how these surfaces compare with many of the common natural and synthetic polymers used as substrates. The second section reviews current work on CNT toxicity, with specific emphasis on neural cell culture systems. Finally, the third section of this paper examines the application of CNT-based materials to directing the differentiation of neural progenitor, mesenchymal and embryonic stem cells.

\section{Carbon nanotubes as neural growth substrates}

Neurons and neural glial cells are often studied in the laboratory using conventional cell culture techniques that have been modified to suit neural cells. However, neurons generally do not attach well to conventional tissue culture substrates, such as polystyrene, and for this reason the culture surface is often modified with charged groups or biomolecules. One strategy is to coat surfaces with natural extracellular matrix proteins found in the nervous system. Such specialized matrix proteins are known to support neural cell survival and proliferation through specific cell-matrix interactions [24]. One of the most widely used natural proteins for neural cell culture is laminin, a multifunctional glycoprotein present in a variety of tissues, including nerve and brain. Laminin has been used to pattern substrates and electrodes to improve neural attachment and extension [25]; however, it can be difficult and expensive to isolate and subsequently reconstitute natural proteins for use in cell culture.

A variety of synthetic polymer alternatives have also been used for neural cell culture. These materials can 


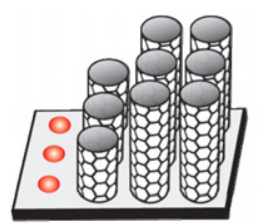

Catalyst

Patterning

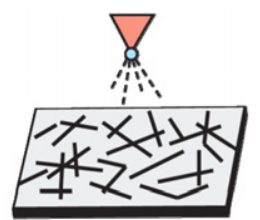

Spray

Coat
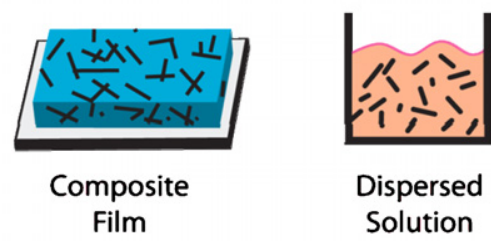

Figure 3. CNT can be deposited onto surfaces in a number of different ways. Patterning of Ni and Fe catalyst particles allows CNT to be grown directly on a substrate in specified patterns. Spray coating produces uniformly absorbed CNT networks with controllable roughness and thickness. Composite films are fabricated by doping polymeric and natural protein matrices with CNT, which can be spin coated onto surfaces or absorbed layer by layer. CNT solutions can also be created by covalently modifying nanotubes and/or coating them with surfactants to improve dispersion stability.

be tailored to promote cell adhesion and growth and, in contrast to naturally derived proteins, can be manufactured in relatively large quantities with high consistency [26]. Polyethyleneimine (PEI) and poly-L-ornithine (PLO) are commonly used polymers that promote neural cell attachment. Both polyethylenedioxythiophene (PEDOT) and polypyrrole (PPy) have also been used as neural growth substrates, and are of particular interest for coatings on neural interface devices due to their high electrical conductivity [27-30]. However, many synthetic and conductive polymers are fabricated using complex or toxic polymerization schemes that are not well suited to cell contacting applications, and these materials are still limited in their effectiveness when compared to natural factors [31].

CNT-based materials have the potential to provide the benefits of both natural and synthetic polymers as substrates for neural cell culture, as well as possessing additional advantages. Compared to other conductive polymers, CNT have a much higher electron mobility potential [32], and therefore theoretically much higher conductivities [33]. In addition, CNT have chemically inert surfaces that can be specifically modified to promote desired cell responses. Recent advances in manufacturing and processing have made large quantities of high-quality CNT available at decreasing cost. There has been an increasing interest in creating composite materials that combine the advantages of native proteins or synthetic polymers with the remarkable properties of CNT, both in 2D [34, 35] and 3D [36, 37] applications. The following section examines how CNT can be used as culture substrates in order to promote neural cell attachment, spreading and function.

\section{CNT culture substrates}

As illustrated in figure 3 , surfaces can be modified with CNT in a number of ways, including direct CNT growth, spray coating and film deposition [38-40]. The use of culture surfaces modified with CNT was one of the first examinations of nanotube-cell interactions, and it has been shown that CNT surfaces are able to support the attachment and growth of numerous cell types, including fibroblasts [41], osteoblasts [42] and cardiac cells [43]. The growth of neural cell types on CNT substrates is a rapidly expanding area of research. The effects of nanoscale materials [44] and conductive substrates $[45,46]$ have been shown to be beneficial to neural cell attachment and growth. Surface topography also plays a major role in determining cellular response, and CNT can be produced in a variety of length scales that can be used to create variations in substrate roughness [47]. The unique physical and electrical properties of CNT materials make them very promising as neural culture surfaces.

Pristine (i.e. purified but unprocessed) CNT have been shown to support the growth of many different cell types. Garcia et al (2007) cultured fibroblasts, Schwann cells and dorsal root ganglia (DRG) on top of pristine MWNT sheets [48]. Fibroblasts were found to attach and proliferate in longterm culture on the MWNT sheets. DRG were also cultured successfully on MWNT sheets with abundant ganglion cell migration. Cultured neurons displayed normal axonal growth, morphology and growth cones. Zhou et al (2007) grew pyrrolized carbon films in pristine form on silicon wafers to act as substrates for the growth of different neural cell lines including mice neuroblastoma, carcinoma (P-19) and rat pheochromocytoma (PC-12) cells [49]. In these studies, carbon surfaces promoted longer and more abundant neurites per cell than control samples.

Unmodified CNT are difficult to use in cell-contacting applications because of their poor solubility in aqueous solutions and the existence of potentially toxic metallic impurities and amorphous carbon residue in such nanotube preparations. In order to improve solubility and cell attachment, modified CNT with a variety of surface charges have been investigated, in efforts to produce more compatible and homogenous cell culture substrates [50]. Hu et al (2004) prepared MWNT that exhibited a range of surface charges from neutral (pristine nanotubes) to negative (carboxylated nanotubes) and positive (ethylenediamine coated), as well as zwitterionic (poly-m-aminobenzene sulfonic acid coated) [51]. Compared to control substrates, hippocampal neurons grown on MWNT substrates exhibited a substantially lower number of neurites, growth cones and neurite branches. Zwitterionic and positively charged MWNT films showed small increases in growth cone number and branching, compared to negative and neutral films. This study showed the effect that charge can have on neural cell morphology, though it is apparent that both the nanotube type and the cell type will affect their interactions. In general, cell lines have proven more hardy and likely to attach to substrates, compared to primary neurons. 
The passive conductance of CNT makes them attractive as substrate coatings for neural culture; however, the effects of conductivity on neural cell growth are not fully understood. Malarkey et al (2009) compared cell culture substrates coated with PEI to surfaces coated with hydrophilic SWNTpolyethylene glycol (PEG) films [52]. The conductivity of the SWNT-PEG coatings was varied by changing the film thickness, while maintaining a consistent surface roughness. It was shown that thinner, less conductive SWNT films resulted in longer neurite processes, while thicker, more conductive films produced larger cell bodies, compared to controls. The smooth, positively charged PEI substrates resulted in a larger number of growth cones per cell body. It is clear from this study that differences in passive conductance, roughness and surface charge can modulate neuronal cell growth and morphology.

Modification of the surface of CNT with bioactive molecules can be used to elicit specific responses from neural cells. Mattson et al (2000) found that pristine MWNT substrates allowed hippocampal neuron attachment but did not support marked neurite outgrowth [53]. However, when MWNT were coated with 4-hydroxynonenal (4-HNE), large increases in the number of neurites per cell and overall neurite length were observed. This study suggested that attachment to MWNT was a key factor for the neuronal response, as free 4-HNE did not induce changes in neuron morphology. Matsumoto et al (2007) functionalized MWNT with nerve growth factor (NGF) and brain-derived neurotrophic factor (BDNF) using amine chemistry [54]. DRG neurite outgrowth on modified MWNT was similar to the effect seen in NGF- and BDNF-supplemented media, demonstrating that the covalently attached factors were still bioactive. In a follow-up study, this group examined the effects of MWNT modified with amino groups in combination with soluble NGF on DRG and PC12 cell growth [55]. Both cell types exhibited increased neurite outgrowth in media containing MWNT. This study also showed that mitogen-activated protein kinase (MAPK) pathways were upregulated in the presence of MWNT, which may have implications on both neurite outgrowth and cell survival.

Neural cells can display a complex array of morphologies that are highly sensitive to their local environment. As shown schematically in figure 4, CNT-based cell culture substrates offer the possibility of exposing such cells to a diverse range of stimuli that can be controlled by taking advantage of the unique properties of nanotubes. There are a growing number of studies that demonstrate the importance of such material properties as nanoscale topography, surface charge and bulk electrical conductivity on neural cell growth and function. The use of CNT materials to examine these issues is still relatively new; however, there is high potential to learn more about the specific stimuli that can be used to promote desired neural cell responses.

\section{Patterning of CNT substrates}

CNT substrates can be fabricated into a variety of geometries and patterns. For example, hard and soft lithographic

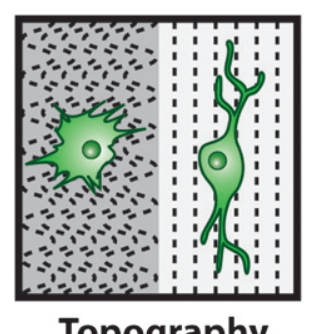

Topography

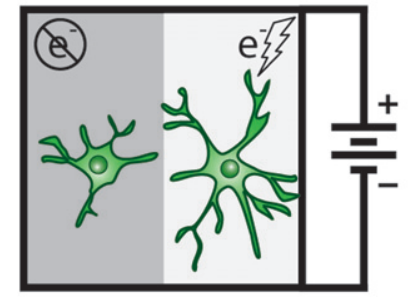

Conductivity
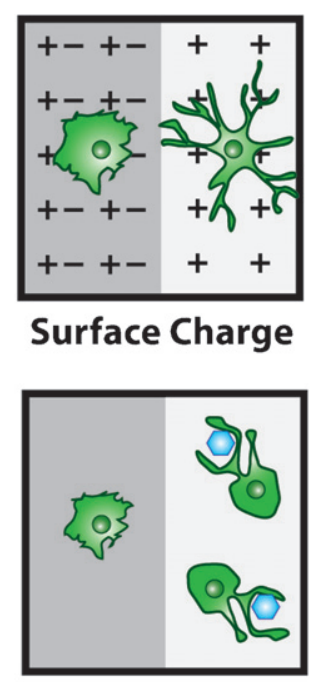

Ligands
Figure 4. CNT substrates can be tailored to exhibit physical, chemical and electrical properties that in turn affect neural cell attachment, growth and function.

methods have been used to etch CNT patterns and deposit CNT solutions [56, 57]. CNT can also be grown directly from patterned catalysts to create aligned templates [58]. These techniques were initially developed to create electrical circuits [59], but more recently have been adapted to building structures for cell culture. In an early study, CorreaDuarte et al (2004) grew cells on 3D structures formed from carboxylated MWNT [60]. Aligned substrates of MWNT were treated with nitric acid to draw upon capillary forces that created pyramid and well structures. These microstructures were able to support the growth, spreading and proliferation of the L929 fibroblast cell line. However, this type of substrate pattern was heterogeneous due to the random nature of its formation, which limited its utility in creating highly defined network patterns.

Substrate patterning has been used to control neural cell adhesion, spreading and growth. It has been shown that the size of patterned stripes and islands is crucial for controlling the migration and spreading of both neurons and glial cells [61]. Zhang et al (2005) examined the effect of cross-hatch, square and spiral MWNT patterns on neurite extension, interaction and guidance [62]. Neurons preferentially adhered to the MWNT patterns, due to the inherent nanoscale roughness. Neuronal growth cones were found to make intimate contact with the nanotube surface, and these strong interactions allowed the neurons to spread along patterns and form interactions with one another. CNTbased substrates could also be used to stimulate and record signals from neural cells. Gabay et al (2005) grew pristine MWNT islands on quartz substrates to probe the growth and electrical activity of rat neural cells [63]. Rat neurons and glial cells grew preferentially on MWNT islands and formed interconnected networks. The neurons were found to be electrophysiologically active with normal resting membrane potentials, demonstrating that the MWNT did not alter the neuronal membrane. 
Neural cell interactions can be further examined by isolating individual cells on patterned microenvironments and studying how they sense their environment and form networks. This type of study has been done using patterned laminin surfaces to examine signaling interactions of small neuron networks [64]. In addition, polydimethylsiloxane (PDMS) templates have been used to examine neuronal cell alignment along channels and protein patterns [65]. Sorkin et al (2009) created direct-growth MWNT patterns on silicon substrates to examine how neurons grow and branch across a heterogeneous culture surface [66]. Neuronal processes tended to wrap and entangle with the rough MWNT islands, whereas smooth surfaces produced straight processes.

The controlled formation of cellular networks has a wide range of applications including the development of high throughput assays [67], biosensors [68] and synthetic neural networks [69]. Understanding how such structures and systems form from neural cells is a major challenge, and studies using patterned CNT can provide insight into how the formation of neural networks might be controlled.

\section{Cell-CNT interactions}

Recreating normal neural cell function depends not only on achieving cell attachment and growth, but also on maintaining appropriate resting membrane potentials and cell signaling. Natural extracellular matrix proteins such as laminin, fibronectin and collagen support neural cell growth, but do not conduct electrical signals, which is a requirement for creating many types of neural devices and probes. Synthetic conductive polymers such as PEDOT and polyaniline (PANI) can be used to create electrically active substrates, but are less conducive to cell attachment than natural proteins. CNT have the potential to provide a substrate that supports cell growth, while also electrically integrating the cells to their substrate. It has been shown that cell-seeded 3D protein materials doped with CNT can conduct electricity [70], and in addition that cellular action can be used to create a directionally conductive matrix [71]. These approaches offer the possibility of creating designer materials that form passively conductive contacts with the resident cells and that transfer signals anisotropically.

The electrical conductivity of CNT can be harnessed to both transmit and receive signals [72] in neural applications. Geith et al (2006) used layer-by-layer deposition to create a SWNT film between two electrode sites [73]. The film was used to simultaneously culture and electrically stimulate NG108 cells, a voltage responsive neuroblastoma cell line. Normal cell differentiation occurred with no adverse effects caused by the SWNT substrate. These materials were used to stimulate the NG108 cells and elicited a current response due to $\mathrm{Na}$ ion influx. This type of system may be used as a tool for understanding the interactions between neuronal cells and other types of cells in co-culture.

Lovat et al (2005) studied hippocampal neurons and astrocytes cultured on MWNT substrates [74]. Cultures on MWNT were stable and exhibited similar morphology and electrical activity, relative to controls grown on a glass substrate. However, as shown in figure 5, neurons on MWNT
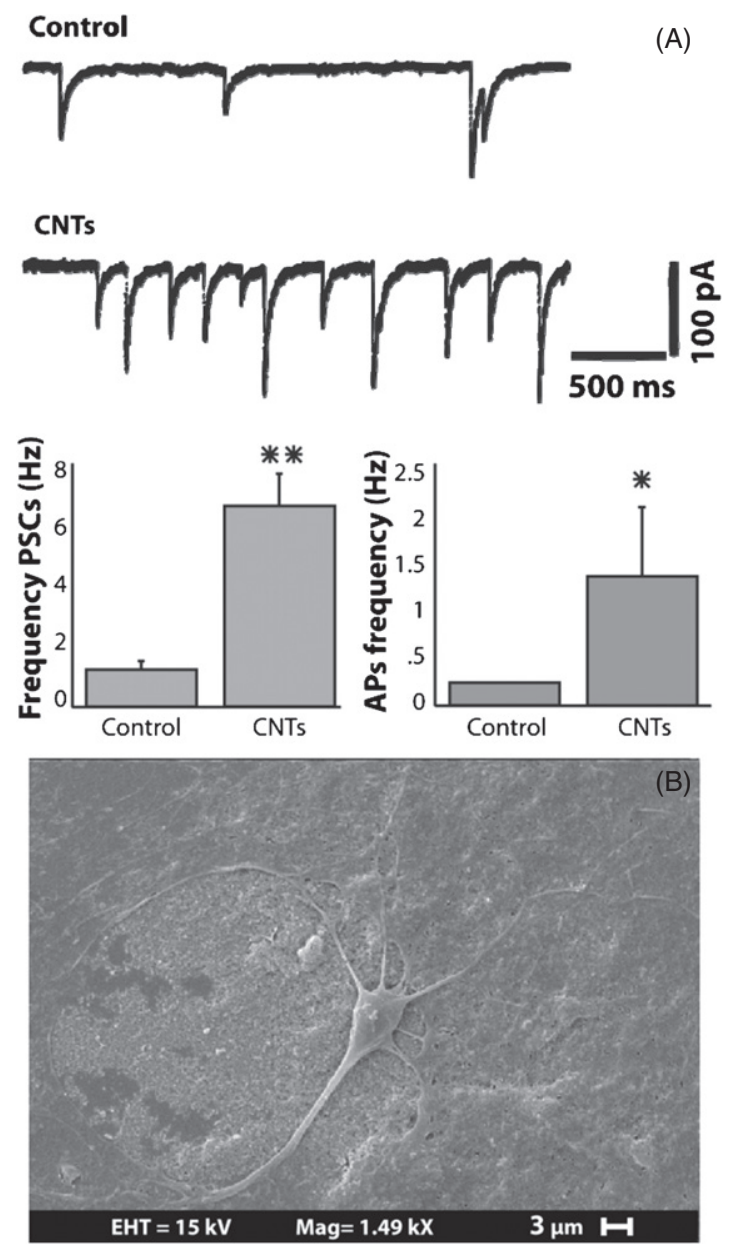

Figure 5. (A) Lovat et al observed a significantly increased frequency of spontaneous post-synaptic currents and action potential firings in neurons grown on MWNT substrates. (B) SEM micrograph showing that hippocampal neurons attach to MWNT substrates and exhibit normal dendrite and axonal spreading. Both panels adapted with permission from [69]. Copyright 2001 American Chemical Society.

substrates exhibited a fourfold increase in the frequency of spontaneous postsynaptic currents and a twofold higher frequency response, compared to glass controls. The cause of these increased response frequencies has not been elucidated, but they have been observed in similar studies in which neurons were stimulated through conductive SWNT layers. A model of the resistive coupling between neurons and SWNT was developed to characterize how neurons might conduct through the substrate [75]. CNT substrates were also compared to non-conductive nanotextured control surfaces [75]. It was observed that neurons grown on SWNT substrates exhibited significantly larger after-potential depolarizations, an indirect effect of dendritic calcium electrogenesis. This phenomenon describes backward-acting action potentials caused by calcium channels in distal regions of the dendrites [76], which may be affected by the presence of SWNT. Taken together, the studies explored here demonstrate the importance of cell-CNT electrical interactions and their use in developing CNT-neuron hybrid systems. 


\section{Biocompatibility of carbon nanotubes}

In general, the interactions between cells and CNT are not well understood. Despite their simple structure and inert chemistry, CNT can be toxic to cells and tissues. CNT toxicity has received a great deal of attention in the past several years, due to increasing attempts to develop CNT-based biomedical technologies [77, 78]. Numerous studies have been performed from a physiological perspective, as well as from the cellular and biomolecular standpoint. However, few clear conclusions have emerged from this body of work to date, due in part to the large degree of inherent variability between the fabrication methods, purity and functionalization of CNT, as well as differences concerning how they are applied to cells and tissues. Therefore, the conclusions that can be drawn from CNT toxicity studies depend heavily on the biocompatibility model used, as well as the purity, concentration and type of CNT being examined.

Early studies on nanotube biocompatibility used animal models to examine the effects of inhalation or intravenous administration of CNT. It was found that inhaled CNT damaged cells near the mucus membrane [79, 80], but when injected intravenously CNT were able to be cleared through the kidneys [81, 82]. In addition, the toxicity of CNT in solution has been assessed at the cellular and molecular levels. However, these studies have varied in their conclusions, with some reports that CNT are essentially benign [83-85] and others that assert CNT have marked toxic effects [86-88]. More recently, the effects of CNT on specific cell types, including neural cells, have been examined. This information is critical for developing new nanotube-based biomedical technologies and for advancing the field of neural engineering.

A variety of strategies have been used to improve the interactions of neural cells with CNT and thereby moderate their cytotoxicity. Non-covalent wrapping has been used to reduce CNT aggregation and improve dispersion. Compared to covalent functionalization, wrapping techniques have the advantage that they maintain the integrity of the carbon lattice and therefore preserve mechanical and electrical properties [89]. Bardi et al (2009) evaluated the cytotoxic effects of MWNT wrapped in Pluronic ${ }^{\circledR}$ F127, a polymer surfactant, on mouse primary cortical neurons and mouse cerebral cortex [90]. It was shown that F127 alone was toxic to cultures of neurons, but that the presence of MWNT negated this effect. Furthermore, F127-wrapped MWNT could be injected into the cerebral cortex of mice without causing degradation of the resident neurons, suggesting that the nanotubes had a protective effect by reducing F127 toxicity.

Belyanskaya et al (2009) also used non-covalently wrapped CNT to examine biocompatibility of nanotube aggregates of varying size on neurons and glial cells [91]. SWNT were coated with the non-ionic surfactant polyoxyethylene sorbitan monooleate (PS80) in such a way as to create larger nanotube aggregates, as well as smaller bundles of nanotubes with improved dispersion. As shown in figure 6, the larger SWNT aggregates resulted in reduced cell number over time. In addition, exposure to SWNT decreased the expression of vimentin, an indication of decreased glial

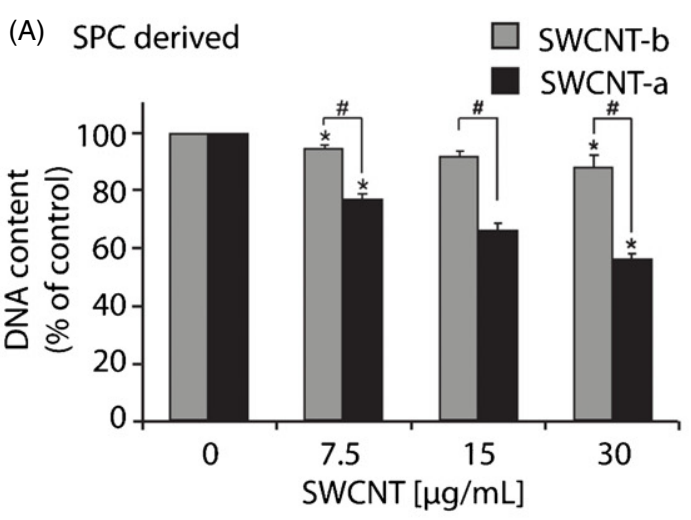

(B) SPC derived

MAP5

Vimentin
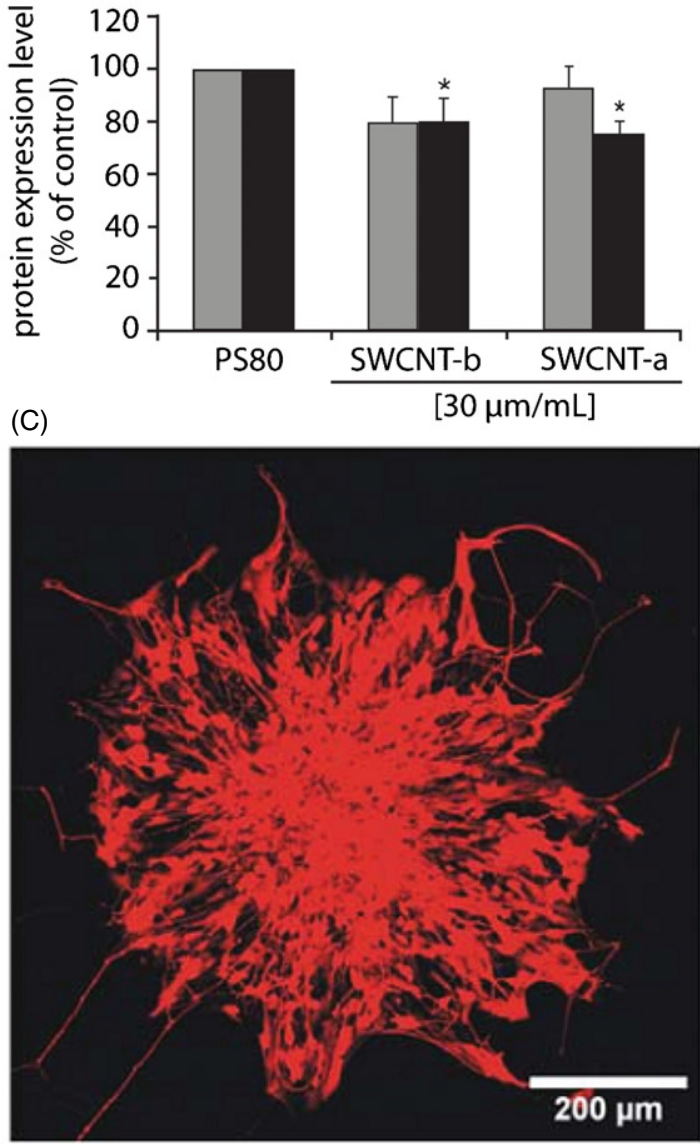

Figure 6. Belyanskaya et al examined the impact of SWNT agglomeration on neuronal toxicity. (A) Larger aggregates (SWCNT-a, $\sim 100 \mathrm{~nm}$ dimension) caused a reduction in cell number compared to smaller bundles (SWCNT-b, $20 \mathrm{~nm}$ ). (B) Both aggregate sizes caused reductions in protein expression. (C) A confocal micrograph shows that spinal cord neuron spreading was unaffected by SWNT (red color shows a ubiquitously expressed protein). All panels adapted with permission from [86]. Copyright 2006 Elsevier.

cell number, but did not affect the expression of the neuronal marker protein MAP5, an indication of neuronal cell number. This study examined the similarities and differences between the responses of both neurons and glial cells to SWNT and suggested that the degree of cytotoxicity is at least partially dependent on the agglomeration state of the nanotubes. 
CNT can also be covalently functionalized in order to permanently modify their structure with specified chemical groups and polymers. Gaillard et al (2009) functionalized MWNT through cycloaddition of azomethine ylides and the addition of specific peptide sequences that promote cell adhesion, including GRGDSP (from fibronectin) and IKVAV (from laminin) [92]. Jurkat tumor cell and splenocyte biocompatibility was analyzed using propidium iodide nuclear staining and flow cytometry. MWNTs functionalized with peptides did not affect cell viability significantly over a range of concentrations from 1 to $100 \mu \mathrm{g} \mathrm{ml}^{-1}$. Hippocampal neurons cultured in contact with modified MWNT displayed typical spontaneous post-synaptic currents, membrane capacitance and resistance. Interestingly, this study localized MWNT to within the intracellular space, suggesting that nanotubes can be internalized by cells without causing toxicity.

Malarkey et al (2008) functionalized SWNT with polyethylene glycol (PEG) and evaluated their cytotoxicity using hippocampal neurons [93]. The nanotubes were added to the culture medium at concentrations ranging from 0.1 to $5.0 \mu \mathrm{g} \mathrm{ml}^{-1}$. Previous work had shown that neurite extensions were fewer but longer when exposed to SWNT-PEG, and it was proposed that CNT interfered with depolarizationdependent plasma membrane/vesicular recycling [94]. A cationic dye that stains cell membranes was used to monitor membrane recycling during and after neuron stimulation in the presence and absence of nanotubes. It was observed that SWNT-PEG conjugates impaired stimulated endocytosis through interaction with the cell membrane, though the mechanism was not specifically investigated.

It is clear that neural cells interact with CNT in a variety of ways, which can be both beneficial and detrimental to cell function. While there is a great need for further investigation of these interactions, the studies performed to date have provided important insights into some of the key factors that affect CNT biocompatibility, including purity, concentration and spatial presentation. The relatively large number of studies that have examined neural cell function on deposited or spray-coated CNT substrates tend to indicate that these materials are not toxic (see e.g. [48, 51, 53, 54, 60, 62, 63]). However, it is difficult to draw hard conclusions based on these studies since in most cases they did not extensively examine cytotoxicity. Most studies that have explicitly examined issues of cytotoxicity have used CNT dispersed in solution. The work reviewed above suggests that CNT aggregation and their interactions with the cell membrane will affect biocompatibility, and in general it is clear that the spatial presentation of CNT is an important determinant of cytotoxic effects. These issues are critical when developing CNT-based neural devices and more work needs to be done in order to fully understand cell-CNT interactions.

\section{Carbon nanotubes for progenitor and stem cell differentiation toward neural lineages}

CNT-based materials have been studied for their ability to promote lineage-specific differentiation of a variety of types of progenitor and stem cells. Controllable microenvironments offer the potential to guide the proliferation and differentiation of stem cells via chemical, electrical and physical signals [95-97]. CNT have high potential in this respect because their surface chemistry, conductance and nanotopography can be varied. It has been shown that a variety of biomaterial substrates can promote neural differentiation of stem cells [98, 99], and CNT may have similar effects if presented appropriately. Understanding how CNT affect neuronal cell differentiation is valuable for a number of potential applications, including improved cell culture substrates, neural probe coatings and nerve guidance conduits.

Mesenchymal stem cells (MSC) are able to differentiate into several specific cell lineages, and this differentiation is affected by the local microenvironment [100]. It has been shown that MSC can become neural-like cells in response to contact with biomaterials and growth factors [101, 102], and that surface chemistry can be modified to promote desired responses [103, 104]. CNT have been examined for this purpose by creating substrates for MSC culture and differentiation. Park et al (2007) used lithography to create SWNT patterns and showed that MSC preferentially attached to the patterns, inducing cell spreading, increased elongation and focal adhesion contacts along the pattern axis [105]. Tay et al (2010) examined how carboxylated SWNT films influenced the behavior of MSC in the absence of a specific differentiation medium [106]. MSC spread and proliferated on SWNT films, and neurogenic markers were upregulated, while osteogenic marker remained low. Focal adhesion distribution was more homogenous in cells grown on the SWNT surface, compared to controls grown on glass. These studies suggest that the nanotopography provided by CNT may play a role in regulating MSC differentiation.

The effects of CNT on MSC differentiation are also dependent on the mode of presentation of the nanotubes, and several studies have examined the effects of dispersed CNT. Mooney et al (2008) evaluated the growth and differentiation of MSC in response to pristine and carboxylated SWNT and MWNT dispersed in cell culture medium [107]. Cell number and metabolic activity were both affected adversely, compared to control cultures. This study also examined markers of the adipogenic, chondrogenic and osteogenic phenotypes, but found no discernable upregulation in these gene families. A similar experiment performed by Liu et al (2010) examined the effect of carboxylated SWNT and MWNT in solution on the proliferation and differentiation of mouse MSC [108]. All CNT formulations caused a significant decrease in viability and proliferative capacity of the stem cells. Osteogenic and adipogenic differentiation were also greatly attenuated by the presence of CNT. The reasons for these changes in response to dispersed CNT are not entirely clear, but these studies suggested that CNT aggregates may interfere with important cell membrane proteins.

Neural stem cells (NSC) are multipotent cells that can differentiate into neurons and glial cells. They are of great interest in neural engineering and regeneration and their interactions with CNT have been studied. Jan et al (2007) compared growth and differentiation of NSC grown on PEIfunctionalized SWNT with those grown on a conventional 
PLO-coated surface [109]. No significant differences in cell viability or attachment were observed, but the SWNTPEI films caused modest induction of neurogenic markers. However, the majority of NSC followed an astrocyte lineage, suggesting that the differentiation stimulus was not strong enough to produce neurons. A related study by Kam et al (2009) examined how SWNT can alter the effects of laminin films on NSC cultures [110]. Laminin/SWNT films supported the growth and proliferation of NSC and also resulted in longer outgrowths, relative to pure laminin substrates. The SWNTcontaining films also supported the differentiation of NSC, including the formation of synapses. Electrical stimulation of the cells through the substrate showed that action potentials were being elicited from the current stimulus. CNT substrates may therefore have utility as coatings for neural probes that support cell growth, promote differentiation and also provide electrical connectivity.

Embryonic stem cells (ESC) offer the potential to create uniform populations of any cell type in the body, including the cells of the brain and nervous system. It has been demonstrated that ESC can be induced to differentiate toward the neural lineage, and there have been early studies investigating CNT materials in this regard. Chao et al (2009) covalently modified CNT with polyacrylic acid (PAA), a negatively charged polymer [111]. The CNT materials supported ESC growth and cell adhesion was higher, compared to PLO and pure PAA control surfaces. Pure PAA resulted in low levels of neural differentiation, whereas ESC grown on PAA-CNT films showed upregulated neurogenic markers and increased branching, indicative of mature neurons. A follow-on study by the same group compared PAAMWNT with pure MWNT, as well as MWNT functionalized with polymethacrylic acid (PMAA) [112]. It was shown that pristine MWNT and functionalized MWNT supported neuron adhesion and differentiation to approximately the same extents, suggesting that these processes are more dependent on the nanotopography of the surface and less on the surface chemistry of the CNT. Sridharan et al (2009) cultured human ESC on surfaces of roughly aligned gelatin, collagen and collagen-SWNT films [113]. ESC cultured on collagen-SWNT gels exhibited significantly higher levels of nestin expression than the other materials and produced long filaments characteristic of ectodermal cells. The increased degree of differentiation was attributed to the physical changes induced by adding SWNT to the collagen film and offers further insight into how CNT can impact the behavior of progenitor and stem cells. More recently, it has also been shown that electrical stimulation can induce neural gene activation and differentiation [114].

Taken together, the studies reviewed above demonstrate the variety of ways in which CNT can be used to impact the adhesion, growth and differentiation of progenitor and stem cells. Such flexible and abundant cell sources are critical to the advancement of neural engineering. It is only recently that nanotechnology and stem cell biology have come together to address some of the challenges in regenerating neural tissues. The use of CNT to control progenitor and stem cell function is an expanding area that may lead to new ways to culture and control the cell populations needed for neural repair.

\section{Conclusions and perspectives}

Recent research activity in the application of CNT to neural engineering has demonstrated that these remarkable materials have great potential to provide important biological insights into neural cell function and control. The ability to vary surface chemistry and nanotopography has revealed information about how neural cells adhere, grow and migrate. In addition, CNT-based materials have garnered interest as a stimulus to promote and direct the differentiation of progenitor and stem cells toward neural lineages. These exciting findings must be tempered with the realization that the cytotoxicology of CNT is still a developing field, and that a better understanding of how CNT interact with the biological environment is required before these materials can be used widely in biomedical applications.

The availability of CNT materials has invigorated a segment of the neural engineering field, in particular those interested in biomaterials-based approaches to sensing and stimulating neural cell signaling [115]. CNT have been used to create novel substrates, patterned surfaces and even 3D structures with properties that cannot be achieved using extracellular matrix proteins or synthetic polymers alone. With respect to neural applications, perhaps the most promising CNT property is high electrical conductivity, which offers the potential to interface directly with functional neurons to both sense and transmit signals [116]. Therefore, CNT can act as both passive and active substrates for use in neural engineering.

Nanotechnology is changing many fields, from electronics to energy production to medicine, though in most cases the potential benefits have not yet fully materialized. Neural engineering has a similar opportunity to move rapidly forward in this area and make key advances that have thus far eluded the field. However, the broad use of CNT and other nanoparticles in neural applications will require a far better understanding of how cells, proteins and nanotubes interact. This knowledge is required to facilitate efficient development of CNT-based neural technologies and to ensure that the diagnostic or therapeutic products that result are both safe and effective. If such knowledge can be obtained, then materials based on CNT can have an important impact on the development of nextgeneration neural implants and devices, as well as providing new approaches to neural tissue engineering and regeneration.

\section{Acknowledgments}

The authors are grateful to their colleagues in the field of nanotube science and tissue engineering for their valuable insights and discussions. We apologize to those whose work could not be included because of space limitations.

\section{References}

[1] Breuer O and Sundararaj U 2004 Polym. Compos. 25 630-45

[2] Avouris P 2002 Acc. Chem. Res. 35 1026-34

[3] Prato M, Kostarelos K and Bianco A 2008 Acc. Chem. Res. 41 60-8

[4] Ajayan P M 1999 Chem. Rev. 99 1787-800 
[5] Baughman R H, Zakhidov A A and de Heer W A 2002 Science 297 787-92

[6] Shim M, Shi Kam N W, Chen R J, Li Y and Dai H 2002 Nano Lett. 2 285-8

[7] Sun Y, Fu K, Lin Y and Huang W 2002 Acc. Chem. Res. 35 1096-104

[8] Wei W, Sethuraman A, Jin C, Monteiro-Riviere N and Narayan R J J. Nanosci. Nanotechnol. 7 1284-97

[9] Chen R J, Bangsaruntip S, Drouvalakis K A, Wong Shi Kam N, Shim M, Li Y, Kim W, Utz P J and Dai H 2003 Proc. Natl Acad. Sci. 100 4984-9

[10] Balani K, Anderson R, Laha T, Andara M, Tercero J, Crumpler E and Agarwal A 2007 Biomaterials 28 618-24

[11] Shi X, Hudson J L, Spicer P P, Tour J M, Krishnamoorti R and Mikos A G 2006 Biomacromolecules 7 2237-42

[12] Johansson F, Carlberg P, Danielsen N, Montelius L and Kanje M 2006 Biomaterials 27 1251-8

[13] He W and Bellamkonda R V 2005 Biomaterials 26 2983-90

[14] Fan Y W, Cui F Z, Hou S P, Xu Q Y, Chen L N and Lee I 2002 J. Neurosci. Methods 120 17-23

[15] Shiral Fernando K A, Lin Y and Sun Y 2004 Langmuir 20 4777-8

[16] Katz E and Willner I 2004 Chem. Phys. Chem. 5 1084-104

[17] Kimura T, Ago H, Tobita M, Ohshima S, Kyotani M and Yumura M 2002 Adv. Mater. 14 1380-3

[18] Alexander J K, Fuss B and Colello R J 2006 Neuron Glia Biol. 2 93-103

[19] Gomez N, Lee J, Nickels J and Schmidt C 2007 Adv. Funct. Mater. 17 1645-53

[20] Donaldson K, Aitken R, Tran L, Stone V, Duffin R, Forrest G and Alexander A 2006 Toxicol. Sci. 92 5-22

[21] Pacurari M, Castranova V and Vallyathan V 2010 J. Toxicol. Environ. Health A73 378

[22] Gilmore J L, Yi X, Quan L and Kabanov A V 2008 J. Neuroimmune Pharmacol. 3 83-94

[23] Lacerda L, Bianco A, Prato M and Kostarelos K 2006 Adv. Drug Deliv. Rev. 58 1460-70

[24] Edgar D, Timpl R and Thoenen H 1984 EMBO J. 3 1463-8

[25] Dertinger S K W, Jiang X, Li Z, Murthy V N and Whitesides G M 2002 Proc. Natl Acad. Sci. 99 12542-7

[26] Guimard N K, Gomez N and Schmidt C E Prog. Polym. Sci. 32 876-21

[27] Li D, Wang W, Wang H, Jia X and Wang J 2008 Appl. Surf. Sci. 255 581-4

[28] Lee J Y, Bashur C A, Goldstein A S and Schmidt C E 2009 Biomaterials 30 4325-35

[29] Durgam H, Sapp S, Deister C, Khaing Z, Chang E, Luebben S and Schmidt C E 2010 J. Biomater. Sci. Polym. Ed. 21 1265-82

[30] Abidian M R, Corey J M, Kipke D R and Martin D C 2010 Small 6 421-9

[31] Wan M 2008 Adv. Mater. 20 2926-32

[32] Dürkop T, Getty S A, Cobas E and Fuhrer M S 2004 Nano Lett. 4 35-9

[33] Zhou C, Kong J and Dai H 2000 Appl. Phys. Lett. 761597

[34] Lu Y, Li T, Zhao X, Li M, Cao Y, Yang H and Duan Y Y 2010 Biomaterials 31 5169-81

[35] Terada M, Abe S, Akasaka T, Uo M, Kitagawa Y and Watari F 2009 Dent. Mater. J. 28 82-8

[36] MacDonald R A, Laurenzi B F, Viswanathan G, Ajayan P M and Stegemann J P 2005 J. Biomed. Mater. Res. A 74A 489-96

[37] Kim Y, Kim H, Yun Y S, Bak H and Jin H 2010 J. Nanosci. Nanotechnol. $103571-5$

[38] Ren Z F, Huang Z P, Xu J W, Wang J H, Bush P, Siegal M P and Provencio P N 1998 Science 282 1105-7

[39] Wu Z et al 2004 Science 305 1273-6

[40] Sano M, Kamino A, Okamura J and Shinkai S 2001 Langmuir 17 5125-8
[41] Lobo A, Antunes E, Machado A, Pacheco-Soares C, Trava-Airoldi V and Corat E 2008 Mater. Sci. Eng. C 28 264-9

[42] Zanello L P, Zhao B, Hu H and Haddon R C 2006 Nano Lett. $6562-7$

[43] Garibaldi S, Brunelli C, Bavastrello V, Ghigliotti G and Nicolini C 2006 Nanotechnology 17 391-7

[44] Yang F, Murugan R, Wang S and Ramakrishna S 2005 Biomaterials 26 2603-10

[45] Oren R et al 2004 J. Biomater. Sci. Polym. Ed. 15 1355-74

[46] Blau A, Weinl C, Mack J, Kienle S, Jung G and Ziegler C $2001 \mathrm{~J}$. Neurosci. Methods 112 65-73

[47] Wang Z, Koratkar N, Ci L and Ajayan P M 2007 Appl. Phys. Lett. 90143117

[48] Galvan-Garcia P, Keefer E W, Yang F, Zhang M, Fang S, Zakhidov A A, Baughman R H and Romero M I 2007 J. Biomater. Sci. Polym. Ed. 18 1245-61

[49] Zhou H, Zhou J, Gupta A and Zou T 2007 Int. J. Med. Sci. 8 884-93

[50] Sreekumar T V, Liu T, Kumar S, Ericson L M, Hauge R H and Smalley R E 2003 Chem. Mater. 15 175-8

[51] Hu H, Ni Y, Montana V, Haddon R C and Parpura V 2004 Nano Lett. 4 507-11

[52] Malarkey E B, Fisher K A, Bekyarova E, Liu W, Haddon R C and Parpura V 2009 Nano Lett. 9 264-8

[53] Mattson M P, Haddon R C and Rao A M $2000 \mathrm{~J}$. Mol. Neurosci. 14 175-82

[54] Matsumoto K, Sato C, Naka Y, Kitazawa A, Whitby R L and Shimizu N 2007 J. Biosci. Bioeng. 103 216-20

[55] Matsumoto K, Sato C, Naka Y, Whitby R and Shimizu N 2010 Nanotechnology 21115101

[56] Li J, Papadopoulos C, Xu J M and Moskovits M 1999 Appl. Phys. Lett. 75367

[57] Meitl M A, Zhou Y, Gaur A, Jeon S, Usrey M L, Strano M S and Rogers J A 2004 Nano Lett. 4 1643-7

[58] Wei B Q, Vajtai R, Jung Y, Ward J, Zhang R, Ramanath G and Ajayan P M 2003 Chem. Mater. 15 1598-606

[59] Wei Y Y and Eres G 2000 Appl. Phys. Lett. 763759

[60] Correa-Duarte M A, Wagner N, Rojas-Chapana J, Morsczeck C, Thie M and Giersig M 2004 Nano Lett. $42233-6$

[61] Thompson D M and Buettner H M 2001 Tissue Eng. 7 247-65

[62] Zhang X, Prasad S, Niyogi S, Morgan A, Ozkan M and Ozkan C S 2005 Sensors Actuators B 106 843-50

[63] Gabay T, Jakobs E, Ben-Jacob E and Hanein Y 2005 Physica A 350 611-21

[64] Kuhn T B, Schmidt M F and Kater S B 1995 Neuron $14275-85$

[65] Clark P, Britland S and Connolly P 1993 J. Cell Sci. $105203-12$

[66] Sorkin R, Greenbaum A, David-Pur M, Anava S, Ayali A, Ben-Jacob E and Hanein Y 2009 Nanotechnology 20015101

[67] Hung P J, Lee P J, Sabounchi P, Lin R and Lee L P 2005 Biotechnol. Bioeng. 89 1-8

[68] Gross G W, Rhoades B K, Azzazy H M E and Ming-Chi Wu 1995 Biosens. Bioelectron. 10 553-67

[69] Chang J C, Brewer G J and Wheeler B C 2001 Biosens. Bioelectron. 16 527-33

[70] MacDonald R A, Voge C M, Kariolis M and Stegemann J P 2008 Acta Biomater. 4 1583-92

[71] Voge C M, Kariolis M, MacDonald R A and Stegemann J P 2008 J. Biomed. Mater. Res. A 86A 269-77

[72] Nugent J M, Santhanam K S V, Rubio A and Ajayan P M 2001 Nano Lett. 1 87-91

[73] Gheith M, Pappas T, Liopo A, Sinani V, Shim B, Motamedi M, Wicksted J and Kotov N 2006 Adv. Mater. 18 2975-9 
[74] Lovat V, Pantarotto D, Lagostena L, Cacciari B, Grandolfo M, Righi M, Spalluto G, Prato M and Ballerini L 2005 Nano Lett. 5 1107-10

[75] Mazzatenta A, Giugliano M, Campidelli S, Gambazzi L, Businaro L, Markram H, Prato M and Ballerini L 2007 J. Neurosci. 27 6931-6

[76] Svoboda K, Denk W, Kleinfeld D and Tank D W 1997 Nature 385 161-5

[77] Smart S, Cassady A, Lu G and Martin D 2006 Carbon 44 1034-47

[78] Lam C, James J T, McCluskey R, Arepalli S and Hunter R L 2006 Crit. Rev. Toxicol. 36 189-217

[79] Warheit D B, Laurence B R, Reed K L, Roach D H, Reynolds G A M and Webb T R 2004 Toxicol. Sci. $77117-25$

[80] Poland C A et al 2008 Nat. Nano. 3 423-8

[81] Liu Z, Davis C, Cai W, He L, Chen X and Dai H 2008 Proc. Natl Acad. Sci. 105 1410-5

[82] Yang S, Wang X, Jia G, Gu Y, Wang T, Nie H, Ge C, Wang $\mathrm{H}$ and Liu Y 2008 Toxicol. Lett. 181 182-9

[83] Ding L, Stilwell J, Zhang T, Elboudwarej O, Jiang H, Selegue J P, Cooke P A, Gray J W and Chen F F 2005 Nano Lett. 5 2448-64

[84] Bottini M, Bruckner S, Nika K, Bottini N, Bellucci S, Magrini A, Bergamaschi A and Mustelin T 2006 Toxicol. Lett. 160 121-6

[85] Cherukuri P, Bachilo S M, Litovsky S H and Weisman R B 2004 J. Am. Chem. Soc. 126 15638-9

[86] Carrero-Sánchez J C, Elías A L, Mancilla R, Arrellín G, Terrones H, Laclette J P and Terrones M 2006 Nano Lett. 6 1609-16

[87] Porter A E, Gass M, Bendall J S, Muller K, Goode A, Skepper J N, Midgley P A and Welland M 2009 Am. Chem. Soc. Nano 3 1485-92

[88] Crouzier T, Nimmagadda A, Nollert M U and McFetridge P S 2008 Langmuir 24 13173-81

[89] Zhao Y and Stoddart J F 2009 Acc. Chem. Res. 42 1161-71

[90] Bardi G, Tognini P, Ciofani G, Raffa V, Costa M and Pizzorusso T 2009 Nanomedicine 596-104

[91] Belyanskaya L, Weigel S, Hirsch C, Tobler U, Krug H F and Wick P 2009 Neurotoxicology 30 702-11

[92] Gaillard C et al 2009 Adv. Mater. 21 2903-8
[93] Malarkey E B, Reyes R C, Zhao B, Haddon R C and Parpura V 2008 Nano Lett. 8 3538-42

[94] Ni Y, Hu H, Malarkey E B, Zhao B, Montana V, Haddon R C and Parpura V 2005 J. Nanosci. Nanotechnol. 5 1707-12

[95] Ren Y, Zhang H, Huang H, Wang X, Zhou Z, Cui F and An Y 2009 Biomaterials 30 1036-44

[96] Castano H, O'Rear E A, McFetridge P S and Sikavitsas V I 2004 Macromol. Biosci. 4 785-94

[97] Dalby M J, Gadegaard N, Tare R, Andar A, Riehle MO, Herzyk P, Wilkinson C D W and Oreffo R O C 2007 Nat. Mater. 6 997-1003

[98] Teixeira A I, Duckworth J K and Hermanson O 2007 Cell Res. 17 56-61

[99] Xie J, Willerth S M, Li X, Macewan M R, Rader A, Sakiyama-Elbert S E and Xia Y 2009 Biomaterials 30 354-62

[100] Lund A W, Yener B, Stegemann J P and Plopper G E 2009 Tissue Eng. Part B. Rev. 15 371-80

[101] Yim E K, Pang S W and Leong K W 2007 Exp. Cell Res. 313 1820-9

[102] Kim S et al 2008 J. Mater. Sci., Mater. Med. $192953-62$

[103] Qian L and Saltzman W M Biomaterials 25 1331-7

[104] Ho M, Yu D, Davidsion M C and Silva G A 2006 Biomaterials 27 4333-9

[105] Park S et al 2007 Adv. Mater. 19 2530-4

[106] Tay C Y et al 2010 Carbon 48 1095-104

[107] Mooney E, Dockery P, Greiser U, Murphy M and Barron V 2008 Nano Lett. 8 2137-43

[108] Liu D, Yi C, Zhang D, Zhang J and Yang M 2010 Am. Chem. Soc. Nano 4 2185-95

[109] Jan E and Kotov N A 2007 Nano Lett. 7 1123-8

[110] Kam N W S, Jan E and Kotov N A 2009 Nano Lett. $9273-8$

[111] Chao T, Xiang S, Chen C, Chin W, Nelson A, Wang C and Lu J 2009 Biochem. Biophys. Res. Commun. 384 426-30

[112] Chao T, Xiang S, Lipstate J F, Wang C and Lu J $2010 A d v$ Mater. 22 3542-7

[113] Sridharan I, Kim T and Wang R 2009 Biochem. Biophys. Res. Commun. 381 508-12

[114] Yamada M et al 2007 Stem Cells 25 562-70

[115] Cogan S F 2008 Annu. Rev. Biomed. Eng. 10 275-309

[116] Pancrazio J J 2008 Nanomedicine (Lond.) 3 823-30 